\title{
Economía conductual, irracionalidad e incertidumbre en la Unión Europea
}

\author{
BELÉN MIRANDA ESCOLAR ${ }^{\text {a }}$, JOSEFA EUGENIA FERNÁNDEZ ARUFE ${ }^{\text {a }}$ \\ a Universidad de Valladolid. Fctad. de CC.EE. y EE. Avda. Valle de Esgueva, 6, 47011 Valladolid, \\ España.E-mail: belen@eco.uva.es, jfarufe@eco.uva.es
}

\section{RESUMEN}

La toma de decisiones por parte de los individuos y de las instituciones ha sido considerada, bajo los supuestos de la teoría económica convencional, como un proceso racional que se desarrolla en un contexto con información completa. Sin embargo, la realidad ha puesto de manifiesto la excesiva simplicidad y falta de realismo de tales supuestos. Este trabajo analiza el proceso de toma de decisiones, desde una perspectiva teórica, y se plantea hasta qué punto la economía conductual puede ayudarnos a comprender algunos hechos que se han producido recientemente, que tiñen de incertidumbre la construcción europea y que ponen en tela de juicio algunos instrumentos tradicionales de la política económica.

Palabras clave: Economía conductual, incertidumbre, irracionalidad, Unión Europea.

\section{Behavioral Economics, Irrationality, Uncertainty and European Union}

\begin{abstract}
The decision-making process on the behalf of a person and institutions has been considered, under the economic conventional assumption, as a rational process developed in a complete information context. However, reality has proved the excessive simplicity and the lack of realism of such assumption. This paper analyzes the decision-making process from a theoretical perspective and it asks if behavioral economics can help us to understand some recent events that suppose uncertainty in the European architecture and question some traditional instruments of economic policy.
\end{abstract}

Keywords: Behavioral Economics, Uncertainty, Irrationality, European Union.

Clasificación JEL: D81, D87, I38

Artículo recibido en octubre de 2017 y aceptado en diciembre de 2017

Artículo disponible en versión electrónica en la página www.revista-eea.net, ref. ə-36119 


\section{INTRODUCCIÓN}

La célebre frase del escritor estadounidense Stephen R. Covey (1989), "no soy producto de mis circunstancias ${ }^{1}$, soy producto de mis decisiones", que en principio puede parecer demasiado tajante, pone de relieve la importancia de las decisiones que toman los individuos para cambiar el curso de los acontecimientos; un proceso complejo en el que entran en juego multitud de variables y en el que influye una gran cantidad de factores.

El 9 de octubre de 2017, Richard H. Thaler recibía el Premio Nobel de Economía por sus hallazgos en el estudio de los procesos de toma de decisiones, particularmente por su contribución al análisis de la racionalidad limitada ${ }^{2}$, las preferencias sociales y la ausencia de autocontrol. Sus trabajos entrelazan la Economía y la Psicología y caen de lleno en el campo de la economía conductual, también denominada economía del comportamiento, una corriente que pone en cuestión el supuesto clásico de que los agentes económicos son racionales.

Este Premio se asemeja al Nobel concedido en 2016 a Oliver Hart y Bengt Holmström por sus aportaciones al proceso de toma de decisiones de las dos partes firmantes de un contrato. Los autores profundizan en los problemas que generan las situaciones con información asimétrica y en los mecanismos que utilizan las partes para reducir este tipo de incertidumbre.

Que el Premio Nobel haya recaído en los dos últimos años en economistas que han dedicado su vida y su trabajo a descifrar los entresijos de la toma de decisiones $^{3}$ quizá no haya sido mera casualidad. Tal vez sea el reconocimiento de que economistas y policy-makers fracasaron al estimar la complejidad del comportamiento humano y de los sistemas en los que vivimos y nos relacionamos.

\footnotetext{
${ }^{1}$ Puede recordarse también la conocida frase de José Ortega y Gasset (1914), “yo soy yo y mi circunstancia, y si no la salvo a ella no me salvo yo", que el filósofo recoge en su obra Meditaciones del Quijote. En ese “yo” pueden esconderse los animal spirits.

2 Este concepto ya fue desarrollado por Herbert Simon (1957b:279) para explicar que las personas intentan buscar una mínima satisfacción, es decir, tratan de alcanzar ciertos niveles de éxito para después, poco a poco, ir ajustando esa solución. Esta estructura de pensamiento, que Simon denominó racionalidad limitada (bounded rationality), estimuló muchos trabajos posteriores sobre el comportamiento de los individuos, las organizaciones y la sociedad.

${ }^{3}$ En 1978 Herbert Simon recibió el Premio Nobel de Economía por su contribución al estudio del proceso de toma de decisiones: "el proceso de toma de decisiones es un proceso mental de racionalidad limitada” (Simon, 1957b:56). Para que el proceso sea racional son necesarias dos condiciones: (i) que se disponga de toda la información relevante en todo momento en las mismas condiciones que la competencia u otros sujetos afectados y (ii) que se cuente con una racionalidad ilimitada que permita valorar correctamente todas las alternativas y elegir la que sea óptima. Desafortunadamente, ambas condiciones brillan por su ausencia en la mayoría de los casos. Lo normal en la realidad son situaciones en las que no se dispone de información completa y en las que tampoco es posible conocer todas las variables implicadas y sus interacciones ni definir correctamente las consecuencias de cada curso de acción.
} 
Siempre que Occidente ha atravesado una crisis se han puesto en tela de juicio los postulados económicos propios de la época. Hasta que la Gran Recesión de 2008 hiciera su aparición, las principales corrientes de pensamiento supieron convivir con menor o mayor fortuna y reconocimiento por parte de la comunidad científica. Sin embargo, tras sus devastadores efectos todo esto cambió. Algunos economistas, entre ellos Krugman (2009:193), han detectado en el sistema una profunda grieta que requiere ser tapada y para ello han recurrido a la economía conductual.

La economía conductual aplica la investigación científica a las tendencias cognitivas y emocionales para una mejor comprensión del proceso de toma de decisiones económicas y sus efectos sobre los precios de mercado, los beneficios y la asignación de recursos (Camerer et al., 2004). Aunque su origen puede encontrarse en la obra The Theory of Moral Sentiments que escribió Adam Smith (1759) sobre los principios psicológicos y los sentimientos morales que rigen la conducta humana, lo cierto es que comenzó a cobrar protagonismo a mediados del siglo XX, a raíz de los modelos sobre utilidad esperada y utilidad descontada en la toma de decisiones bajo condiciones de incertidumbre y consumo intertemporal. Probablemente, la publicación más relevante sea el artículo titulado Prospect Theory: An Analysis of Decision under Risk (Kahneman y Tversky, 1979 ) en el que los autores explicaban las anomalías en la toma de decisiones económicas racionales a través de técnicas de la psicología cognitiva.

En opinión de Krugman (2009:194), la nueva economía debe combinar planteamientos tanto de la economía conductual como del keynesianismo y propone las siguientes pautas para los economistas: (i) hacer frente a la incómoda realidad de que los mercados financieros distan significativamente de la perfección pues "están sometidos a falsas ilusiones extraordinarias y a las locuras de mucha gente"; (ii) admitir que la economía keynesiana es la única solución posible para dar respuesta a las recesiones y depresiones, y (iii) esforzarse para incorporar las realidades de las finanzas a la macroeconomía.

A su vez, Shiller (2016) se cuestiona si el individuo, en su esfera privada, y el Estado, en la pública, a través de sus funciones de regulación y estabilización, han aprendido a predecir futuras crisis económicas y financieras, así como a combatirlas de manera más eficaz, aminorando sus efectos negativos y acortándolos en el tiempo, señalando que la única opción factible para alcanzar estos objetivos sería comprender el papel de los espíritus animales dentro de la economía conductual.

Ambos autores parecen querer colocar las emociones y los sentimientos (culpa, vergüenza, indignación, empatía, simpatía, miedo o repugnancia, entre otros) en un lugar destacado de nuestras decisiones. Como si fuera un sistema de alarma, nos indican las situaciones peligrosas o aversivas que deberíamos evitar, y aquellas otras que nos resultan agradables y a las que, por tanto, 
deberíamos aproximarnos. Aunque visto así parece una cuestión sencilla, las emociones son uno de los procesos psicológicos más complejos y difíciles de explicar, como señalan Wenger et al. (1962:3) al afirmar que "casi todo el mundo piensa que sabe lo que es una emoción hasta que intenta definirla; en ese momento, prácticamente nadie afirma poder entenderla".

Las emociones contrastan con la supuesta racionalidad que guía la toma de decisiones del homo economicus, maximizador en sus opciones, racional en sus decisiones y egoísta en su comportamiento. Sin embargo, los agentes económicos a menudo dan muestras de irracionalidad y "se alejan de las criaturas ficticias que pueblan los modelos económicos” (Thaler, 2016:29). Como señala Kahneman (2013:352), "nuestras dos disciplinas parecen estudiar especies diferentes, que el especialista en conducta económica Richard $H$. Thaler bautizaría luego como econos y humanos. Los humanos que los psicólogos conocen no pueden ser tan consistentes y lógicos como los econos. En ocasiones son generosos, y a menudo están dispuestos a aportar algo al grupo al que están adscritos".

Los planteamientos de la economía conductual quizá puedan ayudarnos a comprender algunos hechos que se han producido recientemente, que tiñen de incertidumbre la construcción europea, y que ponen en tela de juicio algunos de los instrumentos convencionales de la política económica.

\section{LA GRAN RECESIÓN Y EL GRAN DESCONCIERTO: LOS ANIMAL SPIRITS Y LA EXUBERANCIA IRRACIONAL}

El siglo XXI nos sorprendió con una crisis de grandes dimensiones, profundidad y alcance que cogió desprevenidos a economistas, políticos, empresarios y a los ciudadanos, en general. Lo que acontecía constituía un reto desafiante y provocador para el pensamiento económico que no fue capaz de predecirla. Se habían olvidado las oscilaciones recurrentes de la economía ${ }^{4}$. El descrédito de la Ciencia Económica y de la profesión estaba servido (Noiville, 2011).

La magnitud del desastre económico y financiero ocurrido a partir del verano de 2007, cuya transmisión al resto del mundo se produce, principalmente, en el tercer trimestre de 2008, nos conduce a reconsiderar la validez de las teorías dominantes y las políticas que se derivaron de ellas. ¿Cómo pudo ocurrir? ¿Por qué las voces discordantes que opinaban sobre el camino hacia la recesión quedaron oscurecidas ${ }^{5}$ ? Son varias las causas que podemos apuntar: la teoría dominante había funcionado hasta el momento (se producía crecimiento y los

\footnotetext{
${ }^{4}$ Estaríamos dentro de los ciclos largos que son los de más difícil solución ya que, generalmente, convulsionan de forma violenta el funcionamiento económico de la sociedad.

${ }^{5}$ Sobre este tema puede consultarse Fernández Arufe (2012).
} 
precios se controlaban); no se percibían las limitaciones de la racionalidad del pensamiento neoclásico; se consideraba que los mercados seguían siendo eficientes; se seguía creyendo en las expectativas racionales; las interconexiones entre el mercado financiero y el real fueron minusvaloradas; y, sobre todo, no se consideró la globalización con las suficientes precauciones.

En un segundo plano, también deben tenerse en cuenta: las promociones de economistas que estudiaron, en las décadas de los setenta y ochenta, en las elitistas Universidades de Harvard, Chicago, Princeton, Stanford y el Massachusetts Institute of Technology, que no recibieron las enseñanzas adecuadas para comprender lo que estaba ocurriendo; los escasos conocimientos financieros de la población y las innovaciones financieras, que usaban una terminología sofisticada y desconocida para definir y etiquetar determinados productos cuyo contenido quedaba oscurecido (los paquetes estructurados escondían bajo esa denominación pomposa valores de pésima calidad); la facilidad que permitía la globalización para su rápida transmisión y la agilidad que proporcionaban las nuevas tecnologías de la comunicación y la información, que fomentaban una confianza sin límites, harían el resto.

Los estudiosos de la Ciencia Económica habían orillado las enseñanzas y las aportaciones que hubiesen ayudado a explicar lo que sucedió y a tomar las medidas adecuadas. J. Bradford Delong (2011), en un artículo publicado en Project Syndicate en abril de 2011, llamaba la atención sobre la respuesta de Larry Summers, Ex Secretario del Tesoro de Estados Unidos y ex asesor de política económica del Presidente Barack Obama, a la pregunta de un columnista del Financial Times sobre si los economistas (académicos) no entendieron la situación que se iniciaba en 2007.

En dicha respuesta recordaba a una serie de economistas cuyas enseñanzas hubieran servido para explicar lo que estaba aconteciendo en los prolegómenos de la crisis financiera. Entre ellos destacaba a: Walter Bagehot, un director de The Economist de mediados del siglo XIX, que publicó en 1873 un libro sobre mercados financieros titulado Lombard Street, cuyo contenido nos hubiese sido de gran utilidad; Hyman Minsky, al que se puede conocer bien por el uso que hizo Charles P. Kindleberger de sus ensayos completos titulados ¿Can It Happen Again?, en su conocida obra Manias, Panics and Crashes: A History of Financial Crises, publicada en 1978. Los tres autores giran en sus aportaciones en torno a una misma idea: en las épocas de estabilidad es donde se gestan las desestabilizaciones ${ }^{6}$, muchas veces debido a factores (institucionales, culturales y psicológicos, entre otros) que no se tienen en cuenta en los modelos teóricos

\footnotetext{
${ }^{6}$ Las épocas normales de crecimiento no plantean problemas; la teoría concuerda con la realidad sin ninguna fisura. Son épocas de relajamiento y euforia, lo que puede permitir un cierto abandono del control del riesgo, como si no fuera posible que acontecieran situaciones fuera de unas coordenadas conocidas y controlables.
} 
pero que conducen a la economía hacia este tipo de fenómenos (Colander et al., 2009:251).

El optimismo o el pesimismo influyen en las decisiones de los individuos más que las expectativas plasmadas en complejos cálculos matemáticos. Son los espíritus animales, esos motivos no económicos y conductas irracionales, los que están detrás de muchas de las decisiones que adoptan los agentes económicos. Pero aceptar los espíritus animales supone atentar contra la racionalidad neoclásica y el homo economicus como conducta del ser humano" "Si resulta difícil cambiar la mentalidad de la gente sobre lo que toman en el desayuno, ya no digamos sobre los problemas a los que se han enfrentado toda su vida. Durante años, los economistas se han resistido con todas sus fuerzas a empezar a basar sus modelos en una caracterización más precisa del comportamiento humano" (Thaler, 2016:35).

Aunque el fenómeno no es nuevo, a raíz de la última crisis, tanto las causas que están detrás de la inestabilidad de los mercados financieros, como las ideas del pensamiento económico que recogen los animal spirits, son objeto de estudio por parte de un gran número de economistas, pero también de psicólogos y sociólogos.

A lo largo del tiempo, las relaciones entre la Economía y la Psicología se han caracterizado más por los desencuentros que por los deseos de colaboración. Este distanciamiento entre ambas ciencias sociales obedece a posturas encontradas. Por un lado, la Psicología no ha disimulado su afán por centrarse en el ámbito de la clínica, preocupada por resolver problemas patológicos e interesada en comprender el proceso a través del cual los individuos adoptan sus decisiones. Por otro, la Economía ha venido mostrando un deseo incondicional por convertirse en una ciencia exacta (Bénassy-Quéré et al., 2017) haciendo caso omiso de los animal spirits, y prestando una mayor atención no tanto en el proceso de toma de decisiones sino en los resultados que se derivan de ellas (Billón Currás, 2002). Ello ha significado que las teorías y los modelos económicos al uso no hayan sido capaces de explicar los factores desencadenantes y catalizadores de las crisis.

En un intento de reconciliación, George A. Akerlof y Robert J. Shiller, ambos Premios Nobel de Economía en 2001 y 2013, respectivamente, publican en 2009 su obra: Animal Spirits. How Human Psychology Drives the Economy, and why it Matters for Global Capitalism. La primera parte la dedican a

\footnotetext{
7 "Durante la década de 1970 surgió una nueva generación de economistas, denominada nueva economía clásica, cuya crítica postulaba que los escasos espíritus animales que conservaba el pensamiento keynesiano eran demasiado insignificantes como para que tuvieran algún peso en la economía (...) Así que, no sin cierta ironía, se recuperó la antigua economía clásica prekeynesiana carente de desempleo involuntario. Los espíritus animales habían quedado relegados al cubo de la basura” (Akerlof y Shiller, 2009:13).
} 
aspectos que consideran intervienen en los animal spirits, como: la confianza; el sentido de la equidad; la corrupción y la mala fe; la ilusión monetaria; y, las historias que es lo que cada cual interioriza o expone para justificar su papel en la vida. En la segunda, los autores responden a ocho preguntas incluyendo cuestiones sumamente interesantes como: las causas del desempleo involuntario; el porqué de las fluctuaciones en los mercados de valores y en el sector inmobiliario; o, la existencia o no de un trade-off entre inflación y desempleo.

El libro Irrational Exuberance, que Shiller publica en solitario en el año 2000, alberga entre sus páginas el concepto de exuberancia irracional que guarda también relación con los espíritus animales. La expresión tiene su origen en un comentario de Alan Greenspan, en 1996, en ese momento Presidente de la Reserva Federal, al referirse a la sobrevaloración del mercado de valores. Más concretamente, se preguntaba cómo averiguar si la exuberancia irracional había elevado en exceso el valor de los activos, refiriéndose a la crisis inmobiliaria de Japón en plena efervescencia por aquel entonces. Sus palabras originaron una inmediata caída de las cotizaciones bursátiles.

La aportación de Shiller es una crítica a la eficiencia de los mercados, en general, y al de capitales, en particular. Su contribución empírica es destacable ${ }^{8}$ y se considera que anunció con bastante claridad tanto la burbuja de internet del año 2000 como la inmobiliaria ${ }^{9}$ de 2007. Sin embargo, no fue suficiente para alertar sobre lo que estaba por llegar. Tampoco lo fue el hecho de que estos activos, que parecían exentos de riesgo, ya habían estado presentes en otras burbujas inmobiliarias muchos años antes, como es el caso de la japonesa ${ }^{10}$, de la cual, parece ser, no fuimos capaces de aprender. Esta burbuja, igual que muchas otras, se produce por comportamientos de imitación, en los que el individuo reproduce acciones y actitudes sin pensar, solo porque lo han hecho otros con anterioridad. Para el cerebro humano es mucho más sencillo imitar comportamientos que crearlos nuevos. Son muchos los casos en los que el individuo actúa influido por el grupo. Así sucedió, por ejemplo, en la crisis de

\footnotetext{
${ }^{8}$ La primera edición de su obra apareció en el año 2000, en plena burbuja de internet. La segunda edición ampliada, en la que se analiza la burbuja inmobiliaria, la madre de todos los males, se publica en 2005, cuando este mercado se encontraba en auge.

9 "La crisis de las hipotecas subprime fue esencialmente psicológica en origen, como ocurre con todas las burbujas. La crisis no fue causada por el impacto de un meteorito ni por la explosión de un volcán. Vino dada por no ser capaces de anticipar riesgos bastantes evidentes; por pecar de una "exuberancia irracional" ante la perspectiva de generar beneficios siempre y cuando uno estuviera dispuesto a aceptar el concepto de una burbuja que no deja de expandirse" (Shiller, 2008:29).

${ }^{10}$ La burbuja financiera e inmobiliaria de Japón se inició en 1980 y duró aproximadamente diez años. Se considera una de las mayores burbujas especulativas de la historia económica moderna.
} 
los tulipanes de Holanda del siglo XVII ${ }^{11}$, en la burbuja "punto.com” de principios del siglo $\mathrm{XXI}^{12}$, o en el fenómeno más actual de los bitcoins.

\section{LA UNIÓN EUROPEA: UN SISTEMA COMPLEJO CON RIESGOS DE DESESTABILIZACIÓN}

Diez años después del estallido de la crisis financiera, la economía mundial, y en particular la europea, vive un momento incierto. Por un lado, la creciente complejidad de los procesos de decisión político-económica, debido a una mayor presencia de los problemas de trade-off (relaciones contradictorias) entre los objetivos fundamentales de la política económica (Costas y Arias, 2015:282; Arias, 2016:169), dificulta a los policy-makers hacer la elección adecuada. Por otro, la complejidad del comportamiento humano y de los sistemas en los que el individuo vive hace más difícil predecir el resultado final de un referéndum (Brexit), de unas elecciones presidenciales (Donald Trump) o de un intento de secesión de una parte de un Estado miembro de la Unión Europea (Cataluña).

La nueva ciencia de la complejidad puede proporcionar conocimiento para predecir cómo se comportan en realidad los grupos de individuos cuando actúan conjuntamente (efecto manada ${ }^{13}$ ) para formar sistemas económicos o sociopolíticos; sistemas que no operan como una serie de acciones y reacciones simples, sino que se retroalimentan, presentan momentos críticos, singularidades, situaciones de emergencia y otras características propias de los sistemas complejos $^{14}$ (OECD, 2017:4).

El sentimiento de pertenencia a un grupo influye a la hora de evaluar los riesgos y las recompensas de las decisiones que se tomen como tal grupo, siendo cada individuo irracionalmente optimista, e incluso irracionalmente perezoso, en el proceso de toma de decisiones. El individuo, presumiblemente racional, intuye que la valoración entre riesgo y recompensa ya ha sido analizada por los primeros que han adoptado un determinado comportamiento y revisada por aquellos otros que les han seguido. Consecuentemente, se va formando de

11 También conocida como “tulipamanía”, el objeto de especulación fueron los bulbos de tulipán, cuyo precio alcanzó niveles desorbitados, dando lugar a una gran burbuja y una crisis financiera. Para Galbraith (1991:42), “jamás algo tan improbable contribuyó en tan sorprendente medida al engaño masivo que aquí estamos tratando".

12 Entre 1997 y 2000, se incrementó ostensiblemente el valor de las empresas relacionadas con internet. Al cabo de un tiempo, muchas de ellas quebraron o dejaron de operar.

${ }^{13}$ El efecto manada es la tendencia de las personas de imitar a los demás. Estos comportamientos, que al principio fueron creados por nuestro cerebro primitivo para aumentar nuestras posibilidades de supervivencia, explican una gran cantidad de conductas sociales que se producen en nuestros días.

${ }^{14}$ Un sistema complejo está compuesto por varias partes interconectadas cuyos vínculos generan información adicional; es decir, posee más información que la suma de la generada por cada una de las partes de forma independiente. 
manera progresiva el conocido "rebaño de ovejas", en el que cada una sigue la misma senda que las demás, sin entrar a valorar el porqué de sus actuaciones. Otra de las razones que justifica el comportamiento en manada es que tendemos a asumir que el grupo, colectivamente, sabe algo que nosotros desconocemos (Bermejo, 2014). Así, seguimos a otros ignorando irracionalmente la información que tenemos y lo que nos conviene como individuos. A ello habría que añadir que los grupos que se comunican regularmente entre sí acaban pensando de manera parecida.

La Unión Europea, que puede considerarse un sistema complejo, se ve sometida actualmente a una serie de riesgos e incertidumbre, algunos de ellos fruto de decisiones y comportamientos irracionales propios de los animal spirits. ¿Qué ha fallado en la Unión Europa para que se haya sembrado la desconfianza entre sus ciudadanos ${ }^{15}$ ? El pistoletazo de salida lo dio el referéndum convocado en algunos Estados miembros para ratificar el texto que los Jefes de Estado o de Gobierno firmaron en Roma el 29 de octubre de 2004 y que recibió el nombre de “Constitución”. El resultado negativo de Francia y los Países Bajos, dos Estados constitutivos de la Comunidad Económica Europea de 1957, supuso un duro golpe en la línea de flotación de la construcción europea. Años después, los errores de diagnóstico de la crisis financiera, las respuestas de solución desde una perspectiva nacional, el retraso en la reacción del Banco Central Europeo dentro de la eurozona y no acometer una unión bancaria a tiempo, el debilitamiento institucional y la escasa capacidad de gestión para solventar los problemas deterioraron el sentimiento de pertenencia al grupo y ocultaron las ventajas de ser parte de él.

A continuación se destacan algunos de los que consideramos más importantes.

\subsection{Riesgos e incertidumbre política}

Si bien es cierto que la Unión Europea viene mostrando síntomas de debilidad en su proyecto político, en especial desde las precipitadas ampliaciones del siglo XXI, y fatiga crónica en sus instituciones, dejando al descubierto déficits de gobernanza, no lo es menos que, más allá de los riesgos económicos, financieros y de seguridad que le amenazan, los riesgos políticos a los que debe hacer frente son de primera magnitud: el despertar del populismo nacionalista de izquierda o derecha con un fuerte sentimiento antieuropeísta, las aspiraciones separatistas de algunas regiones en diferentes Estados miembros

\footnotetext{
${ }^{15}$ Según el Eurobarómetro estándar de primavera 2017 (EB nº 87) la confianza en la Unión Europea muestra una tendencia al alza y está en su nivel más alto desde 2010. Aun así, solo el $42 \%$ de los ciudadanos confía en la Unión Europea (una subida considerable si tenemos en cuenta que en el otoño de 2016 el porcentaje era del 36\% y del 32\% en el de 2015), y el 56\% se muestra optimista sobre su futuro (Comisión Europea, 2017).
} 
(Cataluña en España; Escocia en Reino Unido ${ }^{16}$; Baviera en Alemania; Flandes en Bélgica, "Padania" (pianura padana, llanura del Po) de las regiones de Lombardía, Aosta, Piamonte, Liguria, Véneto y Emilia-Romagna, por un lado, o el caso del Tirol del Sur, por otro, en Italia; y Córcega en Francia, entre otras) o las consecuencias del Brexit ${ }^{17}$, nunca bien ponderadas ni por el Reino Unido ni por el resto de la Unión Europea, son sólo algunos ejemplos.

El Reino Unido sentó un nuevo e incómodo precedente al responder de forma simple (un frívolo e irresponsable referéndum sobre su permanencia en la Unión) a un problema complejo (Mangas, 2016). Los ciudadanos británicos se manifestaron en las urnas el 23 de junio de $2016^{18}$, después de una campaña que alentó los animal spirits, el sentimiento de pertenencia al grupo y el efecto manada. El miedo se adueñó del voto: miedo a tener vecinos inmigrantes, a la influencia de éstos en la sociedad y a que los trabajadores nativos puedan quedarse sin empleo; miedo a que el Reino Unido pierda su identidad nacional; miedo a que su contribución económica al presupuesto de la Unión pueda tener un coste de oportunidad interno demasiado elevado para ser asumido por los británicos más euroescépticos; y, miedo a la pérdida de poder e influencia del Reino Unido, como nación, en un mundo cada vez más globalizado (Fernández Arufe y Miranda, 2017:142). Un ejemplo claro de cómo la influencia de las personas que se encuentran a nuestro alrededor puede causar efectos en nuestra actitud y en la percepción del mundo que nos rodea.

El psicólogo Solomon Asch estudió en los años cincuenta del pasado siglo el enorme poder de la presión social sobre la opinión individual: "el conformismo social no solo es un suicidio intelectual donde perdemos toda habilidad de razonar por nosotros mismos, sino que también nos puede llevar a cometer acciones muy crueles solo porque la mayoría de las personas lo hacen, perdiendo así nuestra individualidad y convirtiéndonos en parte del pensamiento colectivo" (Asch, 1956:68-70).

Con todo, las negociaciones entre el Reino Unido y el grupo de trabajo de la Comisión, con Michel Barnier de jefe de fila, han ido más despacio de lo que a algunos les hubiera gustado y han sido menos duras de lo inicialmente prometido por la primera ministra Theresa May. Aun así, el 29 de marzo de

${ }^{16}$ Londres permitió en 2014 un referéndum sobre la independencia y la mayoría votó en contra. Tras el Brexit, esta cuestión ha vuelto a resurgir. Sin embargo, las encuestas señalan que si se llegara a celebrar otro referéndum en 2018, el resultado sería similar al de 2014.

${ }^{17}$ La eventual salida del Reino Unido de la Unión Europea ha ocasionado un elevado nivel de incertidumbre que se acentuó a partir del momento en que se activó el artículo 50 del Tratado de Lisboa, dando comienzo al proceso negociador del Brexit.

18 El patrón de voto en el referéndum del Brexit reflejó factores económicos, territoriales, demográficos, culturales y psicosociales. Una región con un PIB per cápita bajo, con una elevada proporción de trabajadores de bajo nivel educativo y cualificación y de población mayor de 65 años se mostró más partidaria de no pertenecer a la Unión Europea. 
2017, el Reino Unido notificó oficialmente al Consejo Europeo su intención de abandonar la Unión, de acuerdo con el artículo 50 del Tratado de Unión Europea. Si nada lo impide, su salida se materializará a las 23:00 horas del 29 de marzo de 2019.

\subsection{Riesgos e incertidumbre en las políticas de estabilización}

La inoperancia de la política monetaria, en un contexto de tipos de interés reales negativos o próximos a cero ${ }^{19}$ (el denominado Zero Lower Bound (ZLB)), que obligó al Banco Central Europeo a adoptar medidas no convencionales $^{20}$, si bien con retraso en relación con la Reserva Federal o el Banco de Japón, entre otros, supone un cambio. Pero es que también ha cambiado el modo en que se relacionan las diferentes variables económicas. El desempleo desciende al mismo tiempo que la inflación. La reacción de los precios a los estímulos monetarios no obedece a las pautas del pasado y ello está complicando la tarea de los bancos centrales, sobre todo de aquéllos que tienen encomendado el objetivo de la estabilidad de precios, como el Banco Central Europeo.

Esta inoperancia de la política monetaria ha vuelto a poner la atención en la política fiscal que tendrá que asumir un papel de mayor protagonismo (Roubini, 2016; Blanchard y Summers, 2017). Aunque la política monetaria ultraexpansiva ha permitido sostener el precio de los activos y el crecimiento económico, manteniendo a raya la deflación en la eurozona en los años más duros de la crisis, está llegando al límite. Si bien, Mario Draghi se está mostrando muy cauteloso al anunciar el mensaje de que la retirada de estímulos será lenta y gradual, existen limitaciones técnicas para mantener el ritmo actual de compras de deuda, entre otras razones por la escasez de bonos alemanes disponibles.

La política fiscal asumiría la responsabilidad en la lucha contra las presiones recesivas ya que las medidas restrictivas de los últimos años han contribuido al saneamiento de las finanzas públicas, dejando cierto margen para estimular la demanda desde el presupuesto. Ahora bien, en este contexto en el que conviven elevados niveles de deuda pública con bajos tipos de interés, la política fiscal

${ }^{19}$ El tipo de referencia de las operaciones principales de financiación del Eurosistema, se situó en el 0,00\% desde el 16 de marzo de 2016. En: http://www.ecb.europa.eu/html/index.en.html.

${ }^{20}$ Una vez que el Banco Central Europeo hubo agotado su "munición convencional”, con efectos claramente insuficientes, optó por recurrir: al efecto anuncio (Forward Guidance), la expansión cualitativa (Qualitative Easing) y la expansión cuantitativa (Quantitative Easing). Esta última se inicia en marzo de 2015, con compras de deuda pública soberana por valor de 60.000 millones de euros mensuales. El Consejo de Gobierno, en su reunión de 8 de diciembre de 2016, decidió ampliar su programa de compra de activos como mínimo hasta diciembre de 2017, si bien, a partir de abril, el importe se redujo desde los 80.000 millones mensuales hasta los 60.000. El 26 de octubre de 2017, dicho Consejo acordó reducir las compras de deuda pública y privada no bancaria en la eurozona hasta un volumen de 30.000 millones de euros mensuales a partir de enero de 2018 y durante nueve meses más. 
debe repensarse no sólo como instrumento de estabilización sino también como de la política de deuda pública (Blanchard y Summers, 2017).

El impulso de la inversión pública (selectiva), encaminada a reparar o reemplazar infraestructuras deterioradas, estimularía no sólo la demanda agregada y el empleo, sino también la oferta, contribuyendo al aumento de la productividad y a la mejora de la eficiencia del sector privado. Precisamente el Plan de Inversiones para Europa ${ }^{21}$ (más conocido como Plan Juncker), que nació para crear un entorno favorable a la inversión y apoyar inversiones en la economía real movilizando recursos públicos y privados, tanto en la Unión en su conjunto como a nivel de Estado miembro, camina en esta dirección. En la misma línea se sitúa el documento Towards a Positive Euro Area Fiscal Stance. Supporting public investments that increase economic growth (European Commission, 2016), en el que se reconoce un cambio de actitud de la Comisión Europea en relación a los estímulos fiscales.

Una política monetaria más agresiva, creando la capacidad necesaria para el control de riesgos, un mayor peso de la política fiscal como instrumento de estabilización, una actitud más relajada en relación con la consolidación de la deuda y una regulación financiera más activa, puede ser una combinación acertada para afrontar los futuros shocks que puedan presentarse.

\subsection{Riesgos e incertidumbre en el marco institucional}

La última crisis trajo consigo una serie de cambios en el marco institucional de la Unión Europea ante el mal funcionamiento de algunos mecanismos que habían sido diseñados para épocas de bonanza pero que se mostraban inoperantes en momentos de recesión.

Se pusieron de manifiesto deficiencias en la gobernanza económica y en la supervisión presupuestaria de los Estados miembros que se resolvieron con distintos instrumentos. El Semestre Europeo, el Two-Pack y el Six-Pack, son sólo algunos de ellos. También dejó al descubierto algunos puntos débiles del Pacto de Estabilidad y Crecimiento, provocando cambios tanto en el pilar correctivo (la activación del criterio de deuda y modificación en las condiciones de las sanciones aplicables), como en el preventivo (obligación de alcanzar y mantener unos Objetivos Presupuestarios a Medio Plazo próximos al equilibrio o en superávit y de someter los Programas de Estabilidad y Convergencia a tal fin).

${ }^{21}$ El Plan inicialmente finalizaba en 2018 con una capacidad para desbloquear nuevas inversiones por un valor de 315.00 millones de euros, a través del Fondo Europeo para Inversiones Estratégicas (FEIE), el pilar central del Plan y una iniciativa conjunta de la Comisión Europea y del Grupo Banco Europeo de Inversiones. Sin embargo, el Ecofin decidió prorrogarlo, como mínimo hasta 2020, y elevar su capacidad de financiación hasta los 500.000 millones de euros. 
La Unión Bancaria es otro cambio institucional que nos legó la crisis ${ }^{22}$, un complemento de la Unión Monetaria que asigna a la Unión Europea la responsabilidad de supervisión, resolución y financiación de bancos en apuros y obliga a las entidades de la eurozona a cumplir las mismas normas. Se basa en tres pilares: el Mecanismo Único de Supervisión (MUS) ${ }^{23}$; el Mecanismo Único de Resolución (MUR) ${ }^{24}$; y, un Sistema Único de Garantía de Depósitos Bancarios ${ }^{25}$. Es precisamente en este último donde se encuentran los mayores escollos.

Las medidas para constituir este Sistema se contemplaban en el Informe de los cinco Presidentes para reforzar la Unión Económica y Monetaria. Se alcanzó un acuerdo político en el que se preveía que el Sistema estuviera completado en 2024. Sin embargo, esta fecha clave ha desaparecido de las negociaciones por las reticencias de Alemania, presionada por sus pequeñas cajas de ahorro que quieren mantener sus fondos de garantía de depósitos locales. Dadas las circunstancias, a corto plazo, Europa parece haber renunciado a este tercer pilar de la Unión Bancaria.

No obstante, la Unión Bancaria ha servido para reordenar los sistemas financieros a nivel nacional, mediante fusiones y adquisiciones, pero todavía queda mucho camino por recorrer para conseguirlo a nivel transfronterizo, entre entidades de diferentes Estados miembros. Aún existen algunas barreras que pueden permanecer incluso en una Unión Bancaria completa, como las características de los negocios, la existencia de políticas financieras diferentes, la lengua o los factores culturales (Goncalves y Wolff, 2017).

La crisis también movió los cimientos institucionales de la política monetaria cuestionando la idoneidad de la independencia de los Bancos Centrales, uno de

${ }^{22}$ Junto al Mecanismo Europeo de Estabilidad (MEDE) que consolida y fusiona el Mecanismo Europeo de Estabilización Europea (MEEF) y la Facilidad Europea de Estabilización Financiera (FEEF). Es el principal mecanismo de apoyo para los países de la eurozona con dificultades temporales para conseguir financiación en los mercados financieros debido a sus niveles de deuda. Sus préstamos están garantizados por los accionistas (países de la zona euro).

${ }^{23}$ Se ocupa de la supervisión directa de los 120 bancos de mayor tamaño de la zona euro. Los supervisores nacionales lo harán del resto bajo la responsabilidad del BCE. Los Estados miembro que no pertenezcan a la eurozona también pueden formar parte de él. Empezó a ejercer el 4 de noviembre de 2014.

${ }^{24}$ Garantiza que las quiebras bancarias que puedan producirse en la Unión Bancaria se gestionen con costes mínimos para el contribuyente y la economía real. La decisión final de iniciar la resolución de un banco recae en la Junta Única de Resolución (JUR), plenamente operativa desde enero de 2016.

${ }^{25}$ Las medidas para constituir un Sistema Europeo de Garantía de Depósitos Bancarios se contemplaban en el Informe de los cinco Presidentes para reforzar la Unión Económica y Monetaria. Un mecanismo por el que, en caso de caída de una entidad, el dinero de los depositantes, hasta 100.000 euros por cuenta, quede a salvo y cubierto a nivel comunitario; así, un país agobiado no tendría que hacer frente solo mediante sus propios mecanismos ni se vería castigado por los mercados (Parlamento Europeo, 2017). 
los requisitos imprescindibles para acceder a la moneda única. Estudios recientes (Blancheton, 2016:104; Costas y Arias, 2015:287-288) siembran dudas sobre la pertinencia de mantener el principio de independencia de los bancos centrales, apelando a la imperiosa necesidad de coordinación con otras políticas y a la falta de legitimidad democrática que supone. Por su parte, Blancheton (2016:104) invita a considerar un nuevo modelo de banco central que se ha ido configurando a partir de la última crisis financiera y de la expansión de la deuda soberana en la mayoría de los países desarrollados, de facto menos independiente, que denomina modelo de independencia tácita de baja intensidad (tacit low-degree independence).

Finalmente, otros cambios institucionales pueden abrirse paso en el futuro. Algunas voces se han alzado reclamando la imposición de condiciones adicionales a la convergencia nominal para acceder al euro, como puede ser el nivel de desarrollo de las instituciones financieras. Otros se producirán a partir de los avances que se vayan registrando en la instrumentación de la política macroprudencial.

\section{REFLEXIÓN FINAL}

Aportaciones desde otras ciencias sociales están desafiando los fundamentos más arraigados de la Economía y cuestionando la forma de visualizar diferentes aspectos del comportamiento de las organizaciones, de las instituciones, de las políticas públicas e incluso de los individuos en su interacción con la sociedad. A raíz de ello, se están produciendo algunos cambios. En 2010, el gobierno británico creó el Equipo de Perspectivas Conductuales y, en la actualidad, otros países están configurando equipos cuyo objetivo es incorporar las investigaciones de otras ciencias sociales en la formulación de políticas públicas.

Muchas de las transformaciones que está experimentando la Unión Europea son inevitables e irreversibles. Otras, más difíciles de prever, se producirán de forma inesperada. Restablecer la confianza, promover el consenso y generar un sentimiento de pertenencia al grupo reviste mayor complejidad en una época en la que la información nunca ha sido tan abundante, accesible y, sin embargo, tan difícil de comprender, comunicar y controlar. Esta tendencia se acentuará en el futuro y continuará cambiando la forma en que funcionan los individuos, las sociedades y las democracias. Será necesario crear nuevas herramientas para facilitar el debate público e implicar a los ciudadanos europeos en las decisiones que se adopten para tener bajo control los animal spirits.

La Unión Europea es un proyecto único en el que se han compartido voluntariamente instrumentos de gobierno que eran potestad de los Estados con el objetivo de servir mejor a los intereses del grupo y de cada uno de sus miembros. No siempre ha sido una tarea fácil, ni tampoco perfecta, pero la 
Unión Europea ha demostrado su capacidad de adaptación en un mundo incierto plagado de riesgos.

Somos conscientes de las dificultades de mover una maquinaria de 27 Estados miembros para la solución de los problemas que se planteen en un futuro, pero también lo somos de la necesidad de profundizar en la unión política para seguir avanzando en la construcción europea.

\section{REFERENCIAS BIBLIOGRÁFICAS}

AKERLOF, G.A. Y SHILLER, R.J. (2009). Animal Spirits. How Human Psychology Drives the Economy, and why it Matters for Global Capitalism. Princeton University Press. Versión en español (2009). Animal Spirits. Cómo influye la psicología humana en la economía. Barcelona. Ediciones Gestión 2000.

ARIAS, X.C. (2016). Política económica y riesgo de estancamiento. En: Sanz Gómez (Coord.): 40 años enseñando Economía y Empresa. Facultad de Ciencias Económicas y Empresariales, 1975-2015. Valladolid. Universidad de Valladolid, pp.167-176.

ASCH, S. E. (1956). Studies of Independence and Conformity: A Minority of One Against a Unanimous Majority. Pshychological Monographs, 70 (Whole no. 416).

BENASSY-QUÉRÉ, A.; BLANCHARD, O.J. and TIROLE, J. (2017). "What Role for Economists in Policy-Making?". Les notes du conseil d'analyse économique, no. 42, pp.1-12.

BILLÓN CURRÁS, M. (2002). "Psicología y Economía desde una perspectiva interdisciplinar". Encuentros multidisciplinares, vol. 4, no. 11, pp. 2-10.

BLANCHARD, O.J. y SUMMERS, L. (2017). Rethinking Stabilization Policy. Back to the Future. Peterson Institute for International Economics.

BLANCHETON, B. (2016). "Central bank independence in a historical perspective. Myth, lessons and a new model". Economic Modelling 52 (2016), pp. 101-107.

BERMEJO, P. (2014). Neuroeconomía, Cómo piensan las empresas. (1 ${ }^{\mathrm{a}}$ ed.). Madrid: Colección Acción Empresarial de LID Editorial Empresarial S.L.

CAMERER, C.F.; LOEWENSTEIN, G \& RABIN, M. (2003). Advances in Behavioural Economics. New York: Princeton University Press.

COLANDER, D.; GOLDBERG, M.; HAAS, A.; JUSELIUS, K.; KIRMAN, A.; LUX, T. \& SLOTH, B. (2009). "The Financial Crisis and the Systemic Failure of the Economics Profession". Critical Review, Vol. 21 (2-3), pp. 249-267.

COMISIÓN EUROPEA (2017a). ¿Primavera europea? El último Eurobarómetro estándar muestra un creciente optimismo. http://europa.eu/rapid/press-release_IP-172127_es.htm. [Último acceso: 09-11-2017].

COMISIÓN EUROPEA (2017b). Libro Blanco sobre el futuro de Europa. Reflexiones y escenarios para la Europa de los Veintisiete en 2005. COM(2017) 2025 de 1 de marzo de 2017. Bruxelles/Brussel.

COSTAS, A. y ARIAS, X.C. (2015). Crecimiento, crisis y formación de políticas económicas. En: Toboso, F. y Caballero, G. (Eds.): Democracia y políticas 
económicas. Elaboración, negociación y opciones. Madrid: Editorial Síntesis, pp.267296.

COSTAS, A. y ARIAS, X.C. (2016). Economía Europea ¿Hacia el "estancamiento secular"?. En: Aranda, E. (Dir) y Cuadrado Roura, J.R. y Casares Ripol, F.J. (Colab): Política Económica: Un contexto de crisis, recuperación e incertidumbre. Madrid: Civitas-Thomson Reuters, pp. 95-120.

COVEY, S.R. (1989). The Seven Habits of Highly Effective People. New York: Publisher: Simon \& Schuster.

DELONG, B.J. (2011). "Economics in Crisis". https://www.project-syndicate.org/ commentary/economics-in-crisis?barrier=true [Último acceso: 14-11-2017].

EUROPEAN COMMISSION (2016). Towards a Positive Euro Area Fiscal Stance. Supporting public investments that increase economic growth. European Political Strategy Centre. Strategic Note, Issue 20. https://ec.europa.eu/epsc/sites/epsc/ files/strategic_note_issue_20.pdf [último acceso: 14-11-2017].

FERNÁNDEZ ARUFE, J. E. (2006). La Ciencia Económica y la evolución social. Lección inaugural del curso académico 2006-2007. Valladolid: Universidad de Valladolid.

FERNÁNDEZ ARUFE, J. E. (2012). La Ciencia Económica: Escenarios y Retos Nuevos. Discurso de ingreso en la Academia de Ciencias Sociales y del Medio Ambiente de Andalucía. http://acsyma.es/Documentos/Actos\%20de\%20Ingreso/Josefa\%20Eugenia \%20Fernández\%20Arufe_ingreso.pdf [Último acceso: 14-11-2017].

FERNÁNDEZ ARUFE, J. E. y GARCÍA CRESPO, M. (2009). Ciclos y crisis en economía. En: Bote, V., Escot, L. y Fernández, J. A. (Editores): Pensar como un economista. Homenaje al Profesor Andrés Fernández Díaz. Madrid: Delta Publicaciones.

FERNÁNDEZ ARUFE, J. E. y MIRANDA ESCOLAR, B. (2017). La política económica en un mundo inestable: Repercusiones en la Unión Europea. En: Mancha Navarro, T. (Dir. y Coord.): Política Económica, Economía Regional y Servicios. Madrid: CivitasThomson Reuters, pp.129-154.

GALBRAITH, J.K. (1991). Breve historia de la euphoria financiera. Barcelona: Editorial Ariel.

GONCALVES RAPOSO, I. and WOLFF, G.B. (2017). "How has banking union changed mergers and acquisitions". Bruegel blog post. http://bruegel.org/2017/09/how-hasbanking-union-changed-mergers-and-acquistions/ [último acceso: 19-11-2017].

KAHNEMAN, D. (2013). Pensar rápido, pensar despacio. Barcelona: Círculo de Lectores.

KAHNEMAN, D. \& TVERSKY, A. (1979). "Prospect Theory: An Analysis of Decision under Risk". Econometrica, Vol. 47, No 2, pp .263-292.

KEYNES, J. M. (1970). Teoría general de la ocupación, el interés y el dinero. México. Fondo de Cultura Económica. Versión original (1936). The General Theory of Employment, Interest and Money. En: The Collected Writings of John Maynard Keynes, vol. VII, paperback ed., London: McMillan and Cambridge University Press for the Royal Economic Society.

KRUGMAN, P. (2009). “¿Cómo pudieron equivocarse tanto los economistas?” Apuntes del CENES, vol. 28, no. 47, pp. 185-194. Versión original (2009): "How Did Economics Get it So Wrong?" En: New York Times, 2nd September, 2009. Project Syndicate.

LEWIN, S.B. (1996). "Economics and Psychology. Lessons for Our Own Day From the Early Twentieth Century". Journal of Economic Literature. Vol. XXXIV (September 1996), pp. 1293-1323. 
MANGAS, A. (2016). Un desastre histórico. Oficina de Transferencia de Resultados de Investigación. Universidad Complutense de Madrid. http://www.racmyp.es/R/racmyp/ docs2/3-2016-06-24-2016_06_not15.pdf [Último acceso: 14-11-2017].

NOIVILLE, F. (2011). Soy economista y os pido disculpas. Barcelona: Editorial Deusto.

OECD (2017). Debate the Issues: Complexity and policy making. OECD Insights, OECD Publishing. Paris. http://dx.doi.org/10.1787/9789264271531-en. [Último acceso: 09-112017].

ORTEGA Y GASSET, J. (1914). Meditaciones del Quijote. Madrid: Publicaciones de la Residencia de Estudiantes.

PARLAMENTO EUROPEO (2017). La Unión Bancaria. Fichas técnicas sobre la Unión Europea.http://www.europarl.europa.eu/atyourservice/es/displayFtu.html?ftuld=FTU_4. 2.4.html [último acceso: 15-11-2017].

ROUBINI, N. (2016). "El regreso de la política fiscal". https://www.projectsyndicate.org/ commentary/shift-from-monetary-to-fiscal-policy-by-nouriel-roubini-2016-09/spanish [Último acceso: 17-11-2017].

SHILLER, R.J. (2005). Irrational Exuberance. Segunda Edición. Princeton: Princeton University Press.

SHILLER, R.J. (2008). El estallido de la burbuja. Cómo se llegó a la crisis y cómo salir de ella. Barcelona: Ediciones Gestión 2000.

SHILLER, R.J. (2016). Fighting the Next Global Financial Crisis. Project Syndicate. https://www.project-syndicate.org/commentary/financial-regulation-public-narratives-byrobert-j--shiller-2016-05?barrier=accessreg [Último acceso: 17-11-2017].

SIMON, H. A. (1957a). Administrative Behavior: A Study of Decision-making Processes in Administrative Organization. (2. ${ }^{\mathrm{a}}$ ed.) Roskilde. MacMillan Publishers Ltd.

SIMON, H. A. (1957b). Models of Man: Social and Rational. New York: John Wiley and Sons, Inc.

SIMON, H. A. (1960). The New Science of Management Decision. New York: Harper and Row.

SMITH, A. (1759). The Theory of Moral Sentiments. Glasgow, Scotland. Versión utilizada (6 $6^{\mathrm{a}}$ edición 1790) Soares, S.M. (Ed) (2005). Metalibri Digital Library. https://www.ibiblio.org/ml/libri/s/SmithA_MoralSentiments_p.pdf [Último acceso: 14-112017].

THALER, R.H (2015). The making of Behavioral Economics Misbehaving. New York. W.W. Norton \& Company Inc. Versión en español (2016). Todo lo que he aprendido con la psicología económica. El encuentro entre la economía y la psicología y sus implicaciones para los individuos. Barcelona. Ediciones Deusto.

TRATADO DE LISBOA por el que se modifican el Tratado de la Unión Europea y el Tratado Constitutivo de la Comunidad Europea (2007/C 306/01) (DOUE 17.12.2007).

WENGER, M. A., JONES, F.N and JONES, M.H. (1962). Physiological Pshycology. Holt Rinehart Winston. 
